\title{
Correlation Factor Taking into Account Spherical Harmonics Through Hypergeometric Functions to Calculate Energies of Li-Like Ions
}

\author{
Boubacar Sow*, Malick Sow, Ahmadou Wagué \\ Department of Physics, Atoms Laser Laboratory, University Cheikh Anta Diop, Dakar, Senegal \\ Email address: \\ boubacar1.sow@ucad.edu.sn (B. Sow) \\ ${ }^{*}$ Corresponding author
}

\section{To cite this article:}

Boubacar Sow, Malick Sow, Ahmadou Wagué. Correlation Factor Taking into Account Spherical Harmonics Through Hypergeometric Functions to Calculate Energies of Li-Like Ions. International Journal of Applied Mathematics and Theoretical Physics.

Vol. 6, No. 2, 2020, pp. 26-34. doi: 10.11648/j.ijamtp.20200602.13

Received: May 6, 2020; Accepted: July 14, 2020; Published: July 28, 2020

\begin{abstract}
Calculations of the energy levels of atoms and ions with $Z \leq 10$ are carried out in this paper using a new wave function including a new method to calculate the correlation factor taking into account spherical harmonics through hypergeometric functions to calculate $(1 \mathrm{~s} 22 \mathrm{~s}){ }^{2} \mathrm{~S}^{\mathrm{e}},(1 \mathrm{sns} 2){ }^{2} \mathrm{~S}^{\mathrm{e}},(1 \mathrm{~s} 2 \mathrm{sns}){ }^{2} \mathrm{~S}^{\mathrm{e}}$ and $(1 \mathrm{~s} 2 \mathrm{snp}){ }^{2} \mathrm{P}^{\mathrm{O}}$ states. The calculations concern the total energy, kinetic energy, Coulomb interaction between the atomic nucleus and the three electrons and the Coulomb interaction between electrons. The results that we have obtained confirm that a relatively theoretical procedure could be used for adequate calculations and understanding of electron correlation effects in doubly excited three- electron states. These results are in compliance with some experimental and theoretical data.
\end{abstract}

Keywords: Wave Function Correlated, Spherical Harmonics, Excited States, Correlation Factor

\section{Introduction}

In recent years, the importance of electron-electron interaction has been widely recognized during multiexcitation and multi-ionization processes. The study of doubly excited He-like systems (ideal for tackling the Coulomb problem with three bodies) made it possible to clarify the theoretical discussions related to the energy position of excited levels and to check the reliability of multiconfiguration type calculations [1-4]. Systems with three excited electrons (four-body Coulomb problem) are more difficult to deal with. The study of states with three excited electrons, first carried out on the He- ion after diffusion of electrons on helium atoms [5], made it possible to observe the $\left(2 s^{2} 2 p\right)^{2} \mathrm{P}^{0}$ and $\left(2 s 2 p^{2}\right)^{2} \mathrm{D}^{\mathrm{e}}$ identified at 1 using the theoretical results of Fano and Cooper [6]. Subsequently, it was easier to start from the lithium atom (with three electrons) than to previously capture an electron on $\mathrm{He}[7,8]$. In addition, the lithium atom constitutes an excellent system for study the four-body Coulomb problem. With this atom, the accent was put on the creation of hollow ionic states by ionization- excitation in layer $\mathrm{K}[6,9-11]$. Likewise, the effect of the term $1 / \mathrm{r}_{\mathrm{ij}}$, which describes the electrostatic repulsion between the electrons, is very important: Consider for example the case of the Helium atom. If we neglect it, we find for the fundamental level an energy of $-108.8 \mathrm{eV}$ (2 electrons subjected to $Z=2$ ) instead of $-79 \mathrm{eV}$ for the experimental value (corresponding to the energy of double ionization of this atom). This same calculation leads to an energy value of simple ionization of the atom of $54.4 \mathrm{eV}$ to compare with the experimental value of $24.6 \mathrm{eV}$. We must therefore take this term into account. It's for this reason we have chosen a wave function of lesser terms which mainly takes into account this interaction. According to the calculations, we see that: the radial correlation expectation values $1 / r_{i j}$ of three electron systems increase when $Z$ increases, as is the case for two electron systems [12-15]. The ratios and the differences with respect to the results of Uptal and Talukdar [12] are practically constants. And for the same atomic systems, $1 / \mathrm{r}_{12}$ in the lithium-like ions (3.44 Rydbergs in the case of Li for example) is greater than that of the corresponding helium-like ions (3.2 Rydbergs for $\left.\mathrm{Li}^{+}\right)$. This 
result indicates that the two electrons in the $1 \mathrm{~s}^{2}{ }^{2} \mathrm{~S}^{\mathrm{e}}$ closed shell core are closer to each other compared to the two electrons in the corresponding helium isoelectronic series. This points out that, in the ground state, the electron-electron effects due to the third electron, decreases the probability of the two electrons in the $1 \mathrm{~s}^{2}{ }^{2} \mathrm{~S}^{\mathrm{e}}$ closed shell core to go far away, and far away from each other, when increasing the charge number $\mathrm{Z}$.

\section{Wave Functions and Calculations}

Schrödinger equation for the Li-like atom and its isoelectronic series attracted a considerable amount of effort.

Lithium isoelectronic sequence serve as prototypes for alkali metal atoms and alkaline earth cations [16]. They are a good test for ab-initio calculation methods. Similarly, Hylleraas-type wave functions containing $\mathrm{r}_{\mathrm{ij}}$ factors have been widely used in atomic physics to study the correlation effects between electrons and / or between electrons and the nucleus for bound and scattering states [17]. The first calculations on the fundamental state of lithium were made by Eckart [18], Guillemin and Zener [19], Wilson [20], and James and Coolidge [21, 22].

Since then, the ground state energy of Lithium has been carried out by Weiss [23], Muszynska et al. [24], King et al. [25-27], McKenzie and Drake [28], Luchow and Kleindienst [29], and Yan et al. [30, 31]. All these studies, except that of Weiss [23], use Hylleraas-type wave functions, of several terms ranging from 100 terms [32] to 3502 terms [31]. The use of Hylleraas-type wave functions have led to fairly precise results in various branches of atomic physics. The new special wave functions containing fewer terms used in this work is defined by:

$$
\psi\left(j, k, m, j^{\prime}, \mathrm{k}^{\prime}, \mathrm{m}^{\prime}\right)=\left\{\sum_{\gamma=0}^{\mathrm{N}-\mathrm{L}-1}\left(\mathrm{~N}^{2} \mathrm{r}_{0}^{2} \mathrm{~L}\right)^{\gamma} \sum_{\nu=0}^{\mathrm{n}_{3}-\ell_{3}-1}\left(\mathrm{n}_{3}{ }^{2} \mathrm{r}_{0}^{2} \ell_{3}\right)^{v}\left(\mathrm{C}_{\mathrm{jkm}}+\mathrm{C}_{\mathrm{j}^{\prime} \mathrm{k}^{\prime} \mathrm{m}^{\prime}}\right)\right\} x\left(r_{12}^{J} r_{13}^{K} r_{23}^{M}\right) \mathrm{e}^{-\left(\alpha_{1} r_{1}+\alpha_{2} r_{2}+\alpha_{3} r_{3}\right)}
$$

Where $\mathrm{J}+\mathrm{K}+\mathrm{M} \leq \Omega, \Omega$ is a positive integer number which determines the expansion length $\mathrm{N} ; \alpha_{1}, \alpha_{2}, \alpha_{3}$ are a nonlinear parameter $r_{1}, r_{2}$ and $r_{3}$ are the coordinates of electrons with respect to the nucleus, $\ell_{1}, \ell_{2}, \ell_{3}$ are respectively the orbital angular moments of the three electrons. $C_{j k m}$ and $C_{j}{ }^{\prime} k^{\prime} m$ ' are parameters to be determined, $r_{0}$ is Bohr radius. $L=\ell_{1}+\ell_{2}$,
$\mathrm{N}=\mathrm{n}_{1}+\mathrm{n}_{2} . \quad \mathrm{J}=\mathrm{j}+\mathrm{j}, \quad \mathrm{K}=\mathrm{k}+\mathrm{k}{ }^{\prime}$ and $\mathrm{M}=\mathrm{m}+\mathrm{m}$ '. The resonance parameters are determined by finding a rate of change which is stable with respect for the non-linear parameters $\alpha_{1}, \alpha_{2}, \alpha_{3}$. According to the calculation of correlation coefficients, proposed by Varshalovich D. A et al. [33], we found a new formula that is proposed by Gning et al. [34]:

$$
r_{i j}^{q}=4 \pi \sum_{\ell=0}^{\infty} \frac{1}{2 \ell+1} a_{\ell}^{q}\left(r_{i}, r_{j}\right) \times\left(Y_{\ell_{i}, m_{i}}\left(\Omega_{1}\right) Y_{\ell_{j}, m_{j}}\left(\Omega_{2}\right)\right) \text { For } \mathrm{r}_{\mathrm{i}}<\mathrm{r}_{\mathrm{j}}
$$

With

$$
a_{\ell}^{q}\left(r_{i}, r_{j}\right)=\frac{\left(-\frac{q}{2}\right)_{\ell}}{\left(\frac{1}{2}\right)_{\ell}} r_{j}^{q}\left(\frac{r_{i}}{r_{j}}\right)^{\ell} F\left(\ell-\frac{q}{2},-\frac{1}{2}-\frac{q}{2}, \ell+\frac{3}{2}, \frac{r_{i}^{2}}{r_{j}^{2}}\right)
$$

The hypergeometric wave function defined by Samarskogo [35]

$$
F\left(\ell-\frac{q}{2},-\frac{1}{2}-\frac{q}{2}, \ell+\frac{3}{2}, \frac{r_{i}^{2}}{r_{j}^{2}}\right)=1-\frac{(2 \ell-q)(1+q)}{2(2 \ell+3)} \times \frac{r_{i}^{2}}{r_{j}^{2}}
$$

Replacing equation (4) with its value in equation (3)

$$
a_{\ell}^{q}\left(r_{i}, r_{j}\right)=\frac{\left(-\frac{q}{2}\right)_{\ell}}{\left(\frac{1}{2}\right)_{\ell}} r_{j}^{q}\left(\frac{r_{i}}{r_{j}}\right)^{\ell} \times\left(1-\frac{(2 \ell-q)(1+q)}{2(2 \ell+3)} \times \frac{r_{i}^{2}}{r_{j}^{2}}\right)
$$

So equation (5) becomes

$$
a_{\ell}^{q}\left(r_{i}, r_{j}\right)=\frac{\left(-\frac{q}{2}\right)_{\ell}}{\left(\frac{1}{2}\right)_{\ell}} \times \frac{r_{i}^{\ell}}{r_{j}^{\ell-q+2}} \times\left(r_{j}^{2}-\frac{(2 \ell-q)(1+q)}{2(2 \ell+3)} \times r_{i}^{2}\right)
$$


And remplacing equation (6) in equation (2)

$$
r_{i j}^{q}=4 \pi \sum_{\ell=0}^{\infty} \frac{1}{2 \ell+1} \times \frac{\left(-\frac{q}{2}\right) \ell}{\left(\frac{1}{2}\right) \ell} \times \frac{r_{i}^{\ell}}{r_{j}^{\ell-q+2}} \times\left(r_{j}^{2}-\frac{(2 \ell-q)(1+q)}{2(2 \ell+3)} \times r_{i}^{2}\right) x\left(Y_{\ell_{i}, m_{i}}\left(\Omega_{i}\right) Y_{\ell_{j}, m_{j}}\left(\Omega_{j}\right)\right)
$$

We obtain:

$$
\begin{gathered}
r_{12}^{J}=4 \pi \sum_{\ell=0}^{\infty} \frac{1}{2 \ell+1} \frac{\left(-\frac{J}{2}\right)_{\ell}}{\left(\frac{1}{2}\right)_{\ell}} \times \frac{r_{1}^{\ell}}{r_{2}^{\ell-J+2}} \times\left[r_{2}^{2}-\frac{(2 \ell-J)(1+J)}{2(2 \ell+3)} \times r_{1}^{2}\right] \times\left(Y_{\ell_{1}, m_{1}}\left(\Omega_{1}\right) Y_{\ell 2, m 2}\left(\Omega_{2}\right)\right) \\
r_{13}^{K}=4 \pi \sum_{\ell=0}^{\infty} \frac{1}{2 \ell+1} \frac{\left(-\frac{K}{2}\right)_{\ell}}{\left(\frac{1}{2}\right)_{\ell}} \times \frac{r_{3}^{\ell}}{r_{1}^{\ell-K+2}} \times\left[r_{1}^{2}-\frac{(2 \ell-K)(1+K)}{2(2 \ell+3)} \times r_{3}^{2}\right] \times\left(Y_{\ell_{1}, m_{1}}\left(\Omega_{1}\right) Y_{\ell 3, m 3}\left(\Omega_{3}\right)\right) \\
r_{23}^{M}=4 \pi \sum_{\ell=0}^{\infty} \frac{1}{2 \ell+1} \frac{\left(-\frac{M}{2}\right)_{l}}{\left(\frac{1}{2}\right)_{l}} \times \frac{r_{2}^{\ell}}{r_{3}^{\ell-M+2}} \times\left[r_{3}^{2}-\frac{(2 \ell-M)(1+M)}{2(2 \ell+3)} \times r_{2}^{2}\right] \times\left(Y_{\ell_{2}, m_{2}}\left(\Omega_{2}\right) Y_{\ell 3, m 3}\left(\Omega_{3}\right)\right)
\end{gathered}
$$

Where

$$
Y_{\ell_{i}, m_{i}}\left(\Omega_{i}\right) Y_{\ell_{j}, m_{j}}\left(\Omega_{j}\right)=\sum_{m=-\ell}^{\ell} Y_{\ell_{i}, m_{i}}^{*}\left(\Omega_{i}\right) Y_{\ell_{j}, m_{j}}\left(\Omega_{j}\right)
$$

represent the spherical harmonics of electron $\mathrm{i}$ or $\mathrm{j}$.

Which $\Omega_{i}=\left(\theta_{i}, \varphi_{i}\right), \Omega_{j}=\left(\theta_{j}, \varphi_{j}\right)$ and $\mathrm{i}, \mathrm{j}=1,2,3$. For a given angular momentum $L$, the angular coupling for the three electrons is: $\left(\ell_{1}, \ell_{2}, \ell_{3}\right)=(0,0,0)_{\mathrm{A}}$ for $\mathrm{S}$ states and

$$
Y_{0,0}\left(\Omega_{i}\right) Y_{0,0}\left(\Omega_{j}\right)=\frac{1}{4 \pi}
$$

$\left(\ell_{1}, \ell_{2}, \ell_{3}\right)=(0,0,1)_{\mathrm{A}}(0,1,0)_{\mathrm{B}}$ for P states,

$$
Y_{\ell}\left(\Omega_{i}\right) Y_{\ell}\left(\Omega_{j}\right)=Y_{1,-1}^{*}\left(\Omega_{i}\right) Y_{1,-1}\left(\Omega_{j}\right)+Y_{1,0}^{*}\left(\Omega_{i}\right) Y_{1,0}\left(\Omega_{j}\right)+Y_{1,1}^{*}\left(\Omega_{i}\right) Y_{1,1}\left(\Omega_{j}\right)
$$

by replacing the values of the spherical harmonics of e- $i$ or $j$, we obtain:

$$
Y_{\ell}\left(\Omega_{i}\right) Y_{\ell}\left(\Omega_{j}\right)=\frac{3}{4 \pi}\left(\begin{array}{l}
\sin \theta_{i} \sin \theta_{j} \cos \varphi_{i j} \\
+\cos \theta_{i} \cos \theta_{j}
\end{array}\right)
$$

For $\varphi_{i}=\varphi_{j}$ we have $\cos \varphi_{i j}=1$

$$
Y_{\ell}\left(\Omega_{i}\right) Y_{\ell}\left(\Omega_{j}\right)=\frac{3}{4 \pi}\left(\sin \theta i \sin \theta_{j}+\cos \theta_{i} \cos \theta_{j}\right)
$$

$\left(\ell_{1}, \ell_{2}, \ell_{3}\right)=(0,0,2)_{\mathrm{A}}(0,1,1)_{\mathrm{B}}$ for $\mathrm{D}$ states,

$$
Y_{\ell}\left(\Omega_{i}\right) Y_{\ell}\left(\Omega_{j}\right)=\sum_{m=-\ell}^{\ell} Y_{\ell, m}^{*}\left(\Omega_{i}\right) Y_{\ell, m}\left(\Omega_{j}\right)=\frac{15 \sin \theta_{i}^{2} \sin \theta_{j}^{2}}{16 \pi}-\frac{15 \cos \theta_{i} \sin \theta_{i} \sin \theta_{j}^{2}}{8 \pi}-\frac{\sqrt{75}\left(3 \cos \theta_{i}^{2}-1\right) \sin \theta_{j}^{2}}{2^{9 / 2} \pi}
$$


The non-relativistic Hamiltonian complex of three-electron atomic systems is written in the form (in atomic units):

$$
\hat{H}=-\frac{1}{2} \sum_{i=1}^{3} \Delta_{i}-Z \sum_{i=1}^{3}\left(\frac{1}{r_{i}}\right)+\sum_{\substack{i, j=1 \\ i \prec j}}^{3}\left(\frac{1}{r_{i j}}\right)=\mathrm{T}+\mathrm{C}+\mathrm{W}
$$

In equation (17), $T$ is the kinetic energy; $C$ is the Coulomb interaction between the atomic nucleus and the two electrons and $\mathrm{W}$ is the Coulomb interaction between electrons. Where the Laplacian is written in the form:

$$
\begin{aligned}
\Delta_{\mathrm{i}}=\frac{1}{r_{i}^{2}} \frac{\partial}{\partial r_{i}}\left(r_{i}^{2} \frac{\partial}{\partial r_{i}}\right)+\frac{1}{r_{i}^{2} \sin \theta_{i}} \frac{\partial}{\partial \theta_{i}}\left(\sin \theta_{i} \frac{\partial}{\partial \theta_{i}}\right)+\frac{1}{r_{i}^{2} \sin ^{2} \theta_{i}} \frac{\partial^{2}}{\partial \varphi_{i}^{2}}=\Delta_{\mathrm{r}_{\mathrm{i}}}+\Delta_{\theta_{\mathrm{i}}}+\Delta_{\varphi_{\mathrm{i}}} \\
\left\{\begin{array}{l}
\Delta_{r_{i}}=\frac{1}{r_{i}^{2}} \frac{\partial}{\partial r_{i}}\left(r_{i}^{2} \frac{\partial}{\partial r_{i}}\right) \\
\Delta_{\theta_{i}}=\frac{1}{r_{i}^{2} \sin \theta_{i}} \frac{\partial}{\partial \theta_{i}}\left(\sin \theta_{i} \frac{\partial}{\partial \theta_{i}}\right) \\
\Delta_{\varphi_{i}}=\frac{1}{r_{i}^{2} \sin ^{2} \theta_{i}} \frac{\partial^{2}}{\partial \varphi_{i}^{2}}
\end{array}\right.
\end{aligned}
$$

With

$$
\begin{aligned}
\mathrm{T} & =-\frac{1}{2}\left(\Delta_{1}+\Delta_{2}+\Delta_{3}\right) \\
& =-\frac{1}{2}\left\langle\psi^{*}\left(j, k, m, j^{\prime}, \mathrm{k}^{\prime}, \mathrm{m}^{\prime}\right)\left|\left(\Delta_{1}+\Delta_{2}+\Delta_{3}\right)\right| \psi\left(j, k, m, j^{\prime}, \mathrm{k}^{\prime}, \mathrm{m}^{\prime}\right)\right\rangle \\
\mathrm{C} & =-Z\left(\frac{1}{\mathrm{r}_{1}}+\frac{1}{\mathrm{r}_{2}}+\frac{1}{\mathrm{r}_{3}}\right) \\
& =-Z\left\langle\psi^{*}\left(j, k, m, j^{\prime}, \mathrm{k}^{\prime}, \mathrm{m}^{\prime}\right)\left|\frac{1}{r_{1}}+\frac{1}{r_{2}}+\frac{1}{r_{3}}\right| \psi\left(j, k, m, j^{\prime}, \mathrm{k}^{\prime}, \mathrm{m}^{\prime}\right)\right\rangle \\
& =-Z \iiint d r_{1}^{3} d r_{2}^{3} d r_{3}^{3} \psi^{*}\left(j, k, m, j^{\prime}, \mathrm{k}^{\prime}, \mathrm{m}^{\prime}\right)\left(\frac{1}{r_{1}}+\frac{1}{r_{2}}+\frac{1}{r_{3}}\right) \psi\left(j, k, m, j^{\prime}, \mathrm{k}^{\prime}, \mathrm{m}^{\prime}\right) \\
\mathrm{W} & =\left(\frac{1}{\mathrm{r}_{12}}+\frac{1}{\mathrm{r}_{13}}+\frac{1}{\mathrm{r}_{23}}\right) \\
& =\left\langle\psi *\left(j, k, m, j^{\prime}, \mathrm{k}^{\prime}, \mathrm{m}^{\prime}\right)\left|\left(\frac{1}{r_{12}}+\frac{1}{r_{13}}+\frac{1}{r_{23}}\right)\right| \psi\left(j, k, m, j^{\prime}, \mathrm{k}^{\prime}, \mathrm{m}^{\prime}\right)\right\rangle \\
& =\iiint d r_{1}^{3} d r_{2}^{3} d r_{3}^{3} \psi^{*}\left(j, k, m, j^{\prime}, \mathrm{k}^{\prime}, \mathrm{m}^{\prime}\right)\left(\frac{1}{r_{12}}+\frac{1}{r_{13}}+\frac{1}{r_{23}}\right) \psi\left(j, k, m, j^{\prime}, \mathrm{k}^{\prime}, \mathrm{m}^{\prime}\right)
\end{aligned}
$$

The normalization constant:

$$
\begin{aligned}
N_{j, k, m, j^{\prime}, \mathrm{k}^{\prime}, \mathrm{m}^{\prime}} & =\left\langle\psi\left(j, k, m, j^{\prime}, \mathrm{k}^{\prime}, \mathrm{m}^{\prime}\right) \mid \psi\left(j, k, m, j^{\prime}, \mathrm{k}^{\prime}, \mathrm{m}^{\prime}\right)\right\rangle \\
N_{j, k, m, j^{\prime}, \mathrm{k}^{\prime}, \mathrm{m}^{\prime}} & =\iiint d r_{1}^{3} d r_{2}^{3} d r_{3}^{3} \Psi_{j, k, m, j^{\prime}, \mathrm{k}^{\prime}, \mathrm{m}^{\prime}}^{*} \times \Psi_{j, k, m, j^{\prime}, \mathrm{k}^{\prime}, \mathrm{m}^{\prime}}
\end{aligned}
$$

With 


$$
\iiint d r_{i}^{3}=\int_{0}^{\infty} d r_{i} \int_{0}^{\pi} d \theta_{i} \int_{0}^{2 \pi} d \varphi_{i} r_{i}^{2} \sin \theta_{i}
$$

and $i=1,2,3$.

\section{Results and Discussions}

In our calculations, we set the dimension $\Omega$ to 4 for the $\left(1 \mathrm{~s}^{2} 2 \mathrm{~s}\right){ }^{2} \mathrm{~S}^{\mathrm{e}}$ and $\left(1 \mathrm{~s}^{2} 2 \mathrm{p}\right){ }^{2} \mathrm{P}^{0}$ states and $\alpha_{1}=\alpha_{2}=4.255, \alpha_{3}=5.400$ $\Omega=3$ for the $1 \mathrm{~s} 3 \mathrm{~s}^{2}, 1 \mathrm{~s} 4 \mathrm{~s}^{2}$ states and $\alpha_{1}=6.754, \alpha_{2}=7.350$, $\alpha_{3}=5.403$. Similarly $\alpha_{1}=0.25$ for the $(1 \mathrm{~s} 2 \mathrm{sns}){ }^{2} \mathrm{~S}^{\mathrm{e}}$ states and $\Omega=4$ for the (1s $2 \mathrm{snp}){ }^{2} \mathrm{P}^{0} \alpha_{1}=\alpha_{2}=\alpha_{3}=2.600 . \Omega=3$ for the $(1 \mathrm{~s} 2 \mathrm{sns}){ }^{2} \mathrm{~S}^{\mathrm{e}}$ and (1s2snp) ${ }^{2} \mathrm{P}^{0}(\mathrm{n} \leq 3)$ and $\alpha_{1}=4.250, \alpha_{2}=8.550, \alpha_{3}=7.855$. After having fixed these parameters, we have Based on the processing of the calculations and energy conversion of $1 \mathrm{a}$. $\mathrm{u} .=2 \mathrm{Ry}=27.211385 \mathrm{eV}$ was used.

Table 1 lists and compare our results for the $\left(1 \mathrm{~s}^{2} 2 \mathrm{~s}\right){ }^{2} \mathrm{~S}^{\mathrm{e}}$ and $\left(1 s^{2} 2 p\right){ }^{2} \mathrm{P}^{0}$ states of the lithium-like ions compared to the Hylleraas results (HR), the multiconfiguration Hartree-Fock (MCHF) and configuration interaction (CI).

The lithium ground-state isoelectronic sequence was investigated Perkins [36], and by Ho [17] using Hylleraas coordinates and calculations on the lithium isoelectronic sequences for some low-lying excited $S$ states were carried out by King [37-38].

Chung, Zhu, and Wang [14] calculated isoelectronic sequences for some low-lying excited states using a full core plus correlation (FCPC) method. And I. sakho [46] using Screening Constant by Unit Nuclear Charge (SCUNC). Regarding the total energy, comparison shows that our results agree well with all auteurs.

Table 2, Table 3, Table 4 show the results of targeted energy $\left(1 \mathrm{sns}^{2}\right){ }^{2} \mathrm{~S}^{\mathrm{e}},(1 \mathrm{~s} 2 \mathrm{sns}){ }^{2} \mathrm{~S}^{\mathrm{e}},(1 \mathrm{~s} 2 \mathrm{snp}){ }^{2} \mathrm{P}^{0}$. All result shows the relatively good accuracy obtained for the values of the energies of atomic systems studied in this work. We can notice that the kinetic energy $T$ and the correlation energy between the two electrons $\mathrm{W}$ increase with the nuclear charge number $\mathrm{Z}$, and decrease when the principal quantum number $n$ increases.

These results point out that the electron correlation effects in the doubly excited states induce the decrease of the probability of electrons to be near the nucleus, as mentioned by Arias de Saavedra et al. [39].

Comparisons of our results concerning the total and excitation energies of the $(1 \mathrm{~s} 2 \mathrm{sns}){ }^{2} \mathrm{~S}^{\mathrm{e}}$ and $(1 \mathrm{~s} 2 \mathrm{snp}){ }^{2} \mathrm{P}^{0}(\mathrm{n} \leq 3)$ levels of $\mathrm{Li}$-like ions $(\mathrm{Z} \leq 6)$ with other theoretical values [14] and experimental data $[40,15]$ are given in Tables 5 . Regarding the comparison with the results of Bhatia [14] listed in Table 5, the ratios between the present total energy calculation $\left(\mathrm{E}^{\mathrm{a}}\right)$ and that of Bhatia [14] $\left(\mathrm{E}^{\mathrm{P}}\right)$ are practically constants and equal to $\mathrm{E}^{\mathrm{a}} / \mathrm{E}^{\mathrm{P}} \approx 1.00$. This may indicate the good agreement between the present calculation and the quasiprojection operator results of Bhatia.

In the same way, considering the results quoted in Table 6, it is seen that the energy ratios between the present calculation and that of Erkoç and Jansen [40] and of David and Chung [15] are practically constant and respectively equal to $\mathrm{E}^{\mathrm{c}} / \mathrm{E}^{\mathrm{P}} \approx 1$.

These ratios point out the good agreement between our results and that of Erkoç and Jansen [40] using a density functional theory and that of David and Chung [15] employing the complex rotation method. Therefore, it should be mentioned that our results are most close to the density functional results of Erkoç and Jansen [40].

Table 1. Comparison of our results in the calculation of total energy of the $\left(1 s^{2} 2 s\right)^{2} S^{e}$ and $\left(1 s^{2} 2 p\right)^{2} P^{O}$ doubly excited states in the lithium-like ions. The energies are expressed in Rydbergs.

\begin{tabular}{|c|c|c|c|c|}
\hline Author & Method & Ref & $-E\left(1 s^{2} 2 s\right)^{2} S^{e}$ & $-E\left(1 s^{2} 2 p\right)^{2} P^{O}$ \\
\hline & & $\mathrm{Li}$ & & \\
\hline Present work & & & 14.955490 & 14.8204196 \\
\hline Chung and Zhu & FCPC & [42] & 14.956119 & 14.8203156 \\
\hline Pipin and Bishop & CI-HR & [43] & 14.956120 & 14.8203108 \\
\hline Tong et al. & $\mathrm{MCHF}$ & [44] & 14.956121 & 14.8203062 \\
\hline Yan and Drake & HR & [41] & 14.956120 & 14.8203130 \\
\hline Pestka and Woznicki & CI-HR & $\begin{array}{l}{[45]} \\
\mathrm{Be}^{+}\end{array}$ & 14.9561202 & 14.8203118 \\
\hline Present work & & & 28.650490 & 28.357542 \\
\hline Chung and Zhu & FCPC & [42] & 28.649522 & 28.358664 \\
\hline I. sakho & SCUNC & [46] & 28.650495 & 29.273420 \\
\hline Perkins & HR & [36] & 28.649140 & \\
\hline Но & HR & [17] & 28.649392 & \\
\hline \multirow[t]{2}{*}{ King } & HR & [37] & 28.649520 & \\
\hline & & $\mathrm{B}^{2+}$ & & \\
\hline Present work & & & 46.85536 & 46.56737 \\
\hline Chung and Zhu & FCPC & [42] & 46.84920 & 46.40888 \\
\hline I. sakho & SCUNC & [46] & 46.85548 & 64.29986 \\
\hline Perkins & HR & [36] & 46.84872 & \\
\hline \multirow[t]{2}{*}{ Но } & HR & [17] & 46.84904 & \\
\hline & & $\mathrm{C}^{3+}$ & & \\
\hline Present work & & & 69.56203 & 68.9620343 \\
\hline Chung and Zhu & FCPC & [42] & 69.55101 & 68.9641964 \\
\hline Perkins & HR & [36] & 69.55044 & \\
\hline
\end{tabular}




\begin{tabular}{|c|c|c|c|c|}
\hline Author & Method & Ref & $-E\left(1 s^{2} 2 s\right)^{2} S^{e}$ & $-E\left(1 s^{2} 2 p\right)^{2} P^{0}$ \\
\hline I. sakho & SCUNC & [46] & 69.56245 & \\
\hline \multirow[t]{2}{*}{ Ho } & HR & [17] & 69.550836 & \\
\hline & & $\mathrm{N}^{4+}$ & & \\
\hline Present work & & & 96.76975 & 96.026472 \\
\hline Chung and Zhu & $\mathrm{FCPC}$ & [42] & 96.75378 & 96.022096 \\
\hline Perkins & HR & [36] & 96.75314 & \\
\hline I. sakho & SCUNC & [46] & 96.76951 & \\
\hline \multirow[t]{2}{*}{ Ho } & HR & [17] & 96.75359 & \\
\hline & & $\mathrm{O}^{5+}$ & & \\
\hline Present work & & & 128.476310 & 127.586316 \\
\hline Chung and Zhu & FCPC & [42] & 128.457077 & 127.581463 \\
\hline Perkins & HR & [36] & 128.45638 & \\
\hline I. sakho & SCUNC & [46] & 128.476180 & \\
\hline Но & HR & $\begin{array}{l}{[17]} \\
\mathrm{F}^{6+}\end{array}$ & 128.456870 & \\
\hline Present work & & & 164.68247 & 162.9982479 \\
\hline Chung and Zhu & FCPC & [42] & 164.66066 & 163.6417488 \\
\hline \multirow[t]{2}{*}{ King } & HR & [38] & 164.660672 & \\
\hline & & $\mathrm{Ne}_{\mathrm{e}}^{7+}$ & & \\
\hline Present work & & & 205.3882100 & 203.7658217 \\
\hline Chung and Zhu & FCPC & [42] & 205.3644556 & 204.2026316 \\
\hline I. sakho & SCUNC & [46] & 205.3881396 & \\
\hline King & HR & [38] & 205.364458 & \\
\hline
\end{tabular}

Table 2. Total energy and excitation energy of the $\left(1 \mathrm{sns}^{2}\right)^{2} S^{e}$ doubly excited states of three electron systems up to $Z=10$ (in Ryd). The excitation energies are calculated with respect to the ground state of the corresponding system.

\begin{tabular}{|c|c|c|c|c|c|c|c|c|}
\hline & & & & $-E$ & & & & \\
\hline $\mathbf{Z}$ & 3 & 4 & 5 & 6 & 7 & 8 & 9 & 10 \\
\hline \multicolumn{9}{|c|}{$\left(1 \mathrm{sns}^{2}\right)^{2} \mathrm{~S}^{\mathrm{e}}$} \\
\hline $1 \mathrm{~s} 2 \mathrm{~s}^{2}$ & 10.8104 & 20.0884 & 32.4401 & 48.0874 & 66.5243 & 87.9402 & 112.5540 & 139.7926 \\
\hline $1 \mathrm{~s} 3 \mathrm{~s}^{2}$ & 9.8031 & 17.8987 & 28.2989 & 41.3667 & 55.7858 & 74.5286 & 93.87265 & 118.4367 \\
\hline $1 \mathrm{~s} 4 \mathrm{~s}^{2}$ & 8.9504 & 17.0544 & 26.9076 & 38.0735 & 53.3674 & 68.9687 & 88.8221 & 109.9467 \\
\hline
\end{tabular}

Table 3. Total energies of $(1 s 2 s n s)^{2} S^{e}$ doubly excited states in the Li-like ions. The energies are expressed in rydbergs.

\begin{tabular}{|c|c|c|c|c|c|c|c|c|}
\hline & & & & $-\mathbf{E}$ & & & & \\
\hline $\mathbf{Z}$ & 3 & 4 & 5 & 6 & 7 & 8 & 9 & 10 \\
\hline \multicolumn{9}{|l|}{ ns } \\
\hline $2 \mathrm{~s}$ & 10.7973 & 19.8935 & 32.4376 & 48.0376 & 67.02571 & 87.3235 & 112.9873 & 139.0435 \\
\hline $3 \mathrm{~s}$ & 10.3188 & 18.5437 & 32.4517 & 44.7643 & 61.6572 & 81.2256 & 102.9876 & 128.0236 \\
\hline $4 s$ & 10.0092 & 18.3457 & 29.7732 & 43.5297 & 59.9273 & 78.9217 & 100.6759 & 124.9876 \\
\hline $5 \mathrm{~s}$ & 10.0588 & 18.0281 & 27.4857 & 40.3275 & 57.2329 & 76.8892 & 99.3456 & 122.9843 \\
\hline $6 \mathrm{~s}$ & 10.2201 & 18.2854 & 30.2046 & 42.4675 & 57.4578 & 75.7683 & 97.5678 & 122.6753 \\
\hline $7 \mathrm{~s}$ & 10.8546 & 17.9876 & 29.0276 & 41.4597 & 59.7654 & 76.9745 & 97.4567 & 120.8796 \\
\hline $8 \mathrm{~s}$ & 9.98745 & 16.5498 & 28.9764 & 42.3287 & 58.2345 & 77.0897 & 97.3476 & 119.7654 \\
\hline 9s & 9.9586 & 18.3457 & 29.0146 & 42.3234 & 58.9876 & 76.0345 & 97.0237 & 119.4357 \\
\hline $10 \mathrm{~s}$ & 9.9545 & 18.2904 & 26.9875 & 42.0987 & 58.0675 & 76.4216 & 96.4568 & 119.0345 \\
\hline
\end{tabular}

Table 4. Total energies of $(1 s 2 \mathrm{snp})^{2} P^{O}$ doubly excited states in the Li-like ions. The energies are expressed in rydbergs.

\begin{tabular}{lllllllll}
\hline & \multicolumn{7}{c}{$\mathbf{- E}$} \\
\hline $\mathbf{Z}$ & $\mathbf{3}$ & $\mathbf{4}$ & $\mathbf{5}$ & $\mathbf{6}$ & $\mathbf{7}$ & $\mathbf{8}$ & $\mathbf{9}$ & $\mathbf{1 0}$ \\
\hline $\mathbf{n p}$ & & & & & & & & \\
\hline $2 \mathrm{p}$ & 10.5687 & 19.7765 & 33.0987 & 45.7895 & 65.8976 & 86.9243 & 111.1765 \\
$3 \mathrm{p}$ & 10.3245 & 19.2098 & 31.8765 & 44.0987 & 62.7658 & 82.0985 & 107.4877 & 138.3476 \\
$4 \mathrm{p}$ & 10.2457 & 18.9987 & 30.0756 & 43.4908 & 60.3452 & 79.0983 & 102.4758 & 127.8764 \\
$5 \mathrm{p}$ & 10.1945 & 18.5432 & 29.7457 & 43.2357 & 59.8796 & 78.7895 & 101.8763 & 123.7543 \\
$6 \mathrm{p}$ & 10.0087 & 18.3476 & 29.4356 & 43.1765 & 59.5678 & 78.2187 & 100.0245 & 122.9876 \\
$7 \mathrm{p}$ & 9.9876 & 18.1298 & 29.3457 & 43.0547 & 59.3298 & 77.7865 & 99.3457 & 122.5678 \\
$8 \mathrm{p}$ & 9.7843 & 18.0325 & 29.1045 & 42.9754 & 59.1204 & 77.3495 & 98.7568 & 122.3457 \\
$9 \mathrm{p}$ & 9.6543 & 17.9876 & 29.10134 & 42.5467 & 58.9873 & 77.2175 & 98.2963 & 122.0453 \\
$10 \mathrm{p}$ & 9.5432 & 17.7463 & 28.9457 & 42.3457 & 58.7595 & 77.05473 & 98.1134 & 121.8769 \\
\hline
\end{tabular}


Table 5. Comparison of our results in the calculation of total energy of the $(1 s 2 s n s)^{2} S^{e}$ and $(1 s 2 s n p)^{2} P^{o}(n \leq 3)$ doubly excited states in the lithium-like ions $(Z \leq$ 6). The energies are expressed in Rydbergs.

\begin{tabular}{|c|c|c|c|c|}
\hline & & Present work & Bathia & $\operatorname{Rapport}^{*} \mathbf{E}^{\mathrm{a} / \mathbf{E}^{\mathrm{p}}}$ \\
\hline Systeme & $(1 \mathrm{~s} 2 \mathrm{snl}){ }^{2} \mathbf{L}^{\pi}$ & $-\mathrm{E}(1 \mathrm{~s} 2 \mathrm{sn}){ }^{2} \mathrm{~L}^{\pi}$ & $-\mathrm{E}(1 \mathrm{~s} 2 \mathrm{snl})^{2} \mathrm{~L}^{\pi}$ & \\
\hline \multirow{3}{*}{$\mathrm{Li}$} & $(1 \mathrm{~s} 2 \mathrm{~s} 2 \mathrm{~s})^{2} \mathrm{~S}^{\mathrm{e}}$ & 10.7973 & 10.8090 & 1.001083 \\
\hline & $(1 \mathrm{~s} 2 \mathrm{~s} 3 \mathrm{~s})^{2} \mathrm{~S}^{\mathrm{e}}$ & 10.3188 & 10.2945 & 0.997645 \\
\hline & $(1 \mathrm{~s} 2 \mathrm{~s} 2 \mathrm{p}){ }^{2} \mathrm{P}^{\mathrm{O}}$ & 10.5687 & 10.6214 & 1.004986 \\
\hline \multirow{5}{*}{$\mathrm{Be}^{+}$} & $(1 \mathrm{~s} 2 \mathrm{~s} 3 \mathrm{p}){ }^{2} \mathrm{P}^{\mathrm{O}}$ & 10.3245 & 10.3636 & 1.003787 \\
\hline & $(1 \mathrm{~s} 2 \mathrm{~s} 2 \mathrm{~s})^{2} \mathrm{~S}^{\mathrm{e}}$ & 19.8935 & 20.2459 & 1.017714 \\
\hline & $(1 \mathrm{~s} 2 \mathrm{~s} 3 \mathrm{~s})^{2} \mathrm{~S}^{\mathrm{e}}$ & 18.5437 & 19.1593 & 1.033197 \\
\hline & $(1 \mathrm{~s} 2 \mathrm{~s} 2 \mathrm{p}){ }^{2} \mathrm{P}^{\mathrm{O}}$ & 19.7765 & 19.9138 & 1.006942 \\
\hline & $(1 \mathrm{~s} 2 \mathrm{~s} 3 \mathrm{p}){ }^{2} \mathrm{P}^{\mathrm{O}}$ & 19.2098 & 19.1200 & 0.995325 \\
\hline \multirow{3}{*}{$\mathrm{B}^{2+}$} & $(1 \mathrm{~s} 2 \mathrm{~s} 2 \mathrm{~s})^{2} \mathrm{~S}^{\mathrm{e}}$ & 32.4376 & 32.6829 & 1.007562 \\
\hline & $(1 \mathrm{~s} 2 \mathrm{~s} 3 \mathrm{~s})^{2} \mathrm{~S}^{\mathrm{e}}$ & 32.4517 & 30.6716 & 0.945146 \\
\hline & $(1 \mathrm{~s} 2 \mathrm{~s} 2 \mathrm{p}){ }^{2} \mathrm{P}^{\mathrm{O}}$ & 33.0987 & 32.2058 & 0.973023 \\
\hline \multirow{5}{*}{$\mathrm{C}^{3+}$} & $(1 \mathrm{~s} 2 \mathrm{~s} 3 \mathrm{p}){ }^{2} \mathrm{P}^{\mathrm{O}}$ & 31.8765 & 30.6127 & 0.960353 \\
\hline & $(1 \mathrm{~s} 2 \mathrm{~s} 2 \mathrm{~s})^{2} \mathrm{~S}^{\mathrm{e}}$ & 48.0376 & 48.1209 & 1.001734 \\
\hline & $(1 \mathrm{~s} 2 \mathrm{~s} 3 \mathrm{~s})^{2} \mathrm{~S}^{\mathrm{e}}$ & 44.7643 & 44.7249 & 0.999119 \\
\hline & $(1 \mathrm{~s} 2 \mathrm{~s} 2 \mathrm{p}){ }^{2} \mathrm{P}^{\mathrm{O}}$ & 45.7895 & 47.4970 & 1.037290 \\
\hline & $(1 \mathrm{~s} 2 \mathrm{~s} 3 \mathrm{p}){ }^{2} \mathrm{P}^{\mathrm{O}}$ & 44.0987 & 44.8103 & 1.016136 \\
\hline
\end{tabular}

${ }^{\mathrm{p}}$ Present work.

${ }^{a}$ A. K. Bhatia [14].

Table 6. Comparison of our results in the calculation of total energy of the $(1 s 2 s 2 p)^{2} P^{o}$ level in the lithium-like ions with other literature values. The results are expressed in Rydbergs.

\begin{tabular}{llllll}
\hline $\mathbf{Z}$ & Present work & Erkoç and Jansen & David and Chung & Rapport & Rapport \\
\hline & $-\mathbf{E}^{\mathbf{P}}$ & $-\mathbf{E}^{\mathbf{c}}$ & $-\mathbf{E}^{\mathbf{e}}$ & $\mathbf{E}^{\mathbf{c}} / \mathbf{E}^{\mathbf{p}}$ & $\mathbf{E}^{\mathbf{e} / \mathbf{E}^{\mathbf{p}}}$ \\
\hline 3 & 10.5687 & 10.59664 & 10.73696 & 1.002643655 & 1.015920595 \\
4 & 19.7765 & 19.90236 & 20.13696 & 1.006364119 & 1.018226683 \\
5 & 33.0987 & 32.21030 & 32.54492 & 0.973159066 & 0.983268829 \\
6 & 45.7895 & 47.52198 & 47.96012 & 1.037835748 & 1.047404317 \\
7 & 65.8976 & 65.83988 & 66.38402 & 0.999124095 & 1.007381452 \\
8 & 86.9243 & 87.16696 & 87.81906 & 1.002791624 & 1.010293554 \\
9 & 111.1765 & 111.50664 & 112.2684 & 1.002969512 & 1.009821320 \\
10 & 138.3476 & 138.86268 & 139.7358 & 1.003723085 & 1.010034145 \\
\hline
\end{tabular}

${ }^{\mathrm{P}}$ Present work.

${ }^{\mathrm{c}} \mathrm{S}$. Erkoç and H. J. F. Jansen. [40].

${ }^{\mathrm{e}}$ B. F. David and K. T. Chung. [15].

Figures 1-3 show the plots of the energy $E=f\left(\alpha_{i} ; D\right)$ as a function of the parameter $\alpha_{\mathrm{i}}$ and the dimension D.

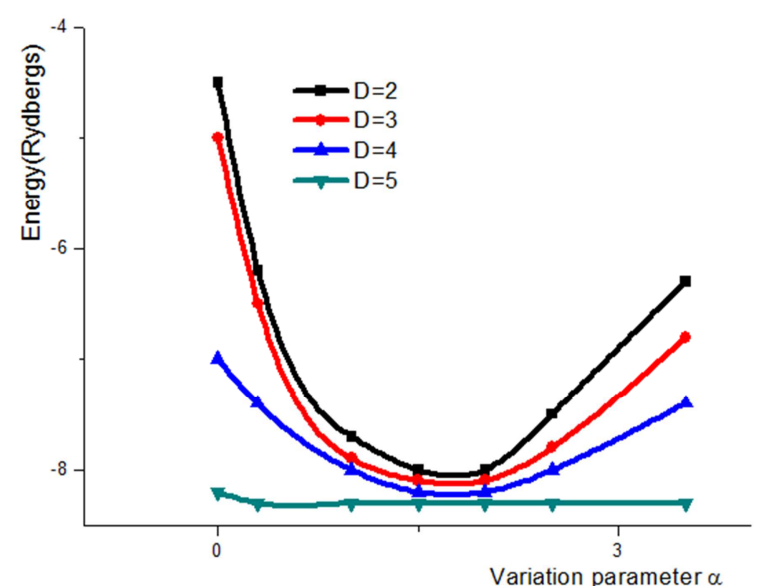

Figure 1. Plots $E=f\left(\alpha_{i}\right)$ of the energy $E$ in terms of the variation parameter $\alpha$ for the dimensions 2, 3, 4, 5 of the Li-like ions $\left(1 s 4 s^{2}\right)^{2} S^{e}$ state. The close similarity of the plots for $D=4$ and $D=5$ shows the convergence of the minima when $D=5$.



Figure 2. Plots $E=f\left(\alpha_{i}\right)$ of the energy $E$ in terms of the variation parameter $\alpha$ for the dimensions 3, 4, 5, 6 of the Li-like ions $(1 s 2 s 2 p){ }^{2} P^{O}$ state. The close similarity of the plots for $D=4$ and $D=5$ shows the convergence of the minima when $D=5$. 


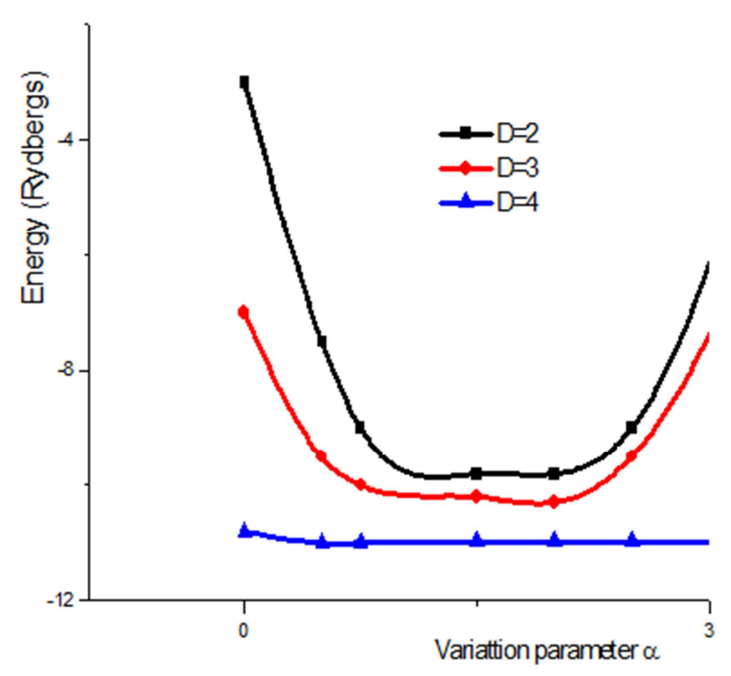

Figure 3. Plots $E=f(\alpha)$ of the energy $E$ in terms of the variation parameter $\alpha$ for the dimensions 2, 3, 4, 6 of the Li-like ions (1s2s2s) ${ }^{2} S^{e}$ state. The close similarity of the plots for $D=4$ shows the convergence of the minima when $D=4$.

These plots concern the lithium atom and are performed for the $\left(1 s 4 s^{2}\right)^{2} \mathrm{~S}^{\mathrm{e}},(1 \mathrm{~s} 2 \mathrm{~s} 2 \mathrm{p}){ }^{2} \mathrm{P}^{\mathrm{O}}$ and $(1 \mathrm{~s} 2 \mathrm{~s} 2 \mathrm{~s}){ }^{2} \mathrm{~S}^{\mathrm{e}}$ states.

A good approximation for the eigenvalues is obtained when the minima of the functions $\left(\mathrm{dE} / \mathrm{d} \alpha_{\mathrm{i}}=0\right)$ converge with increasing values of $\mathrm{D}$ and when the functions exhibit a plateau. As long as the functions exhibit no plateau and as long as the minima of the functions do not converge with increasing values of $\mathrm{D}$, one has not yet found a good approximation.

In our calculations the exhibition of a plateau and the convergence of the minima arise when $D=4$. This could be seen from the figures where there is a close similarity of the plots for $\mathrm{D}=4$ and $\mathrm{D}=5$. This similarity could be explained by the relatively weak contribution of the configurations $(0,0,0)$; $(0,0,1) ;(1,0,0)$ to the calculations of the eigenvalues.

It is important to notice that we have obtained a good approximation for the eigenvalues with a relatively small dimension of the basis $(D=4)$. Using atomic systems with three electrons and using bases as weak as possible for systems, we can observe convergence with very small dimensions. So these results is in good agreement with one aspect of electron correlation and the resemblance of the atomic levels to the rotational spectrum of Li-like ions.

\section{Conclusion}

With a relatively small dimension of the basis functions and using Hylleraas-type wave functions, we have obtained a quantitative and qualitative estimation of electron correlation effects for the ground-state and for doubly excited states of the Lithium-Like ions. It has been demonstrated the possibilities to use a new correlated wave function in the study of $\left(1 s^{2} 2 s\right){ }^{2} S^{e}$, $\left(1 \mathrm{sns}^{2}\right){ }^{2} \mathrm{~S}^{\mathrm{e}},(1 \mathrm{~s} 2 \mathrm{sns}){ }^{2} \mathrm{~S}^{\mathrm{e}}$ and $(1 \mathrm{~s} 2 \mathrm{snp}){ }^{2} \mathrm{P}^{\mathrm{O}}$ doubly excited states in the Lithium-like ions.

Thus, a new correlated wave function is performed and results obtained are shown by the comparison with various available theoretical literature values. One can notice that, the merit of the basis functions is to give the possibilities to calculate accurate energies for doubly excited states in three electron systems using separately the kinetic energy, the Coulomb potential and the Coulomb interaction between electrons. We will also note the fact that the theoretical calculation of the different terms of the disturbance Hamiltonian poses a real complex problem due to the heaviness of the mathematical formalism implemented. One of the merits of this method is to be able to get around these mathematical difficulties by a separate calculation of the different terms of the Hamiltonian which leads to very precise results. Thus our results can be used in a wide field, which goes from the physics of plasmas in intense laser field to atomic and molecular dynamics in femto second regime $\left(1 \mathrm{fs}=10^{-15} \mathrm{~s}\right)$ in infrared (IR). The latter field has experienced considerable development in the last decade, it is in fact linked to the technique of frequency drift amplification (Chirp Pulse Amplification) which generates very short intense pulses in the IR domain; a crucial technological advance which in 2018 won the Nobel Prize in Physics for Gérard Mourou and Donna Strickland.

\section{Acknowledgements}

The authors would like to thank Professor Amlan Kusum Roy Division of Chemical Sciences Indian Institute of Science Education and Research (IISER) -Kolkata for providing us with several papers in this area and for his advice.

\section{References}

[1] Ermolaev M., J. Phys. B 17, 1069 (1984).

[2] Fischer C. F., Comput. Phys. Commun. 64, 369 (1991).

[3] Bronk T., Reading J. F. and Ford A. L., J. Phys. B 31, 2477 (1998).

[4] Godunov A. L., Ivanov P. B., Shipakov V. A., Moretto-Capelle P., Bordenave-Montesquieu D. and Bordenave-Montesquieu A., J. Phys. B 33, 971 (2000).

[5] Kuyatt C. E., Simpson J. A. and Mielczarek S. R. Phys. Rev. 138, A385 (1965).

[6] Fano U. and Cooper J. M., Rev. Mod. Phys. 40, 441 (1968).

[7] Skogvall B., Chesnel J.-Y., Frémont F., Lecler D., Husson X., Lepoutre A., Hennecart D., Grandin J.-P, Sulik B., Salin A. and Stolterfoht N., Phys. Rev. A 51, R4321 (1995).

[8] Diehl S., Cubaynes D., Wuilleumier F. J., Bizau J.-M., Journel L., Kennedy E. T., Blancard C., VoKy L., Faucher P., Hibbert A., Berrah N., Morgan T. J., BozekJ., and Schlachter A. S., Phys. Rev. Lett. 79, 1241 (1997).

[9] Diehl S., Cubaynes D., Zhou H. S., VoKy L., Wuilleumier F. J., Kennedy E. T., Bizau J.- M., Manson S. T., Blancard C., Berrah N. +and Bozek J., J. Phys. B 33, L487 (2000).

[10] Tanis J. A., Chesnel J.-Y., Frémont F., Hennecart D., Husson X., Cassimi A., Grandin J. P., Skogvall B., Sulik B., Bremer J.-H. and Stolterfoht N., Phys. Rev. Lett. 83, 1131 (1999). 
[11] Chesnel J.-Y., Tanis J. A., Sulik B., Skogvall B., Rangama J., Frémont F., Bremer J.-H., Cassimi A., Hennecart D., Hoffmann V., Husson X., Landers A. L. and Stolterfoht N., Photonic, Electronic and Atomic Collisions (XXII ICPEAC) Proceedings ed. J Burgdorfer, J Cohen, S Datz, and C Vane, pp 699-707 (2002).

[12] R. Uptal and B. Talukdar, Phys. Scr. 59, 133 (1999).

[13] T. J. McIlrath, T. B. Lucatorto, and R. P. Madden, Phys. Rev. Lett. 25, 1537 (1970).

[14] A. K. Bhatia, Phys. Rev. A 18, 2523 (1978).

[15] B. F. David and K. T. Chung, Phys. Rev. A 39, 3942 (1989).

[16] Thakkar, A. J., Koga, T., Tanabe, T. and Teruya, H., Chem. Phys. Lett., 366, p. 95-99, (2002).

[17] Ho Y. K., Intern. J. of Quant. Chem., 10, pp. 1077 - 1082, (1981).

[18] Eckart, C., Phys. Rev., 36, 878, (1930).

[19] Guillemin Jr., V. and Zener, C., Z. Phys., 61, 199, (1930).

[20] Wilson Jr. E. B., J. Chem. Phys. 1, 210, (1933).

[21] James, H. M., and Coolidge, A. S., Phys. Rev., 47, 700, (1935).

[22] James, H. M., and Coolidge, A. S., Phys. Rev., 49, 688, (1936).

[23] Weiss, A. W., Phys. Rev., 122, 1826, (1961).

[24] Muszynska, J., Papierowska, D. and Woznicki, W., Chem. Phys. Lett., 76, 136, (1980).

[25] King, F. W. and Shoup, V., Phys. Rev. A., 33, 2940, (1986).

[26] King, F. W., Phys. Rev. A., 40, 1735, (1989).

[27] King, F. W. and Bergsbaken, M. P., J. Chem. Phys., 93, 2570, (1990).

[28] McKenzie, D. K. and Drake, G. W. F., Phys. Rev. A., 44, R6973, (1991).
[29] Luchow, A. and Kleindienst, H., Int. J. Quantum Chem., 51, 211, (1994).

[30] Yan, Z. C. and Drake, G. W. F., Phys. Rev. A 52, 3711, (1995).

[31] Yan, Z. C., Tambasco, M. and Drake, G. W. F., Phys. Rev. A., 57, 1652, (1998).

[32] Larsson, S., Phys. Rev. 169, 49 (1968).

[33] Varshalovich, D. A., et al.,. Quantum Theory of Angular Momentum. Scientific Publishing Co. Ltd. (1988).

[34] Youssou Gning, et al.. Radiation Physics and Chemistry 106, $1-$ 6 (2015).

[35] Samarskogo, A.. Edition Science, Moscow, 281 p. (1974).

[36] J. F. Perkins, Phys. Rev. A 13, 915 (1976).

[37] F. W. King, Phys. Rev. A 38, 6017 (1988).

[38] F. W. King, Phys. Rev. A 40, 1735 (1989).

[39] F. Arias de Saavedra, I. Porras, E. Buendia, F. J. Galvez, J. Phys. B 28, 3123 (1995).

[40] S. Erkoç and H. J. F. Jansen, Phys. Rev. A 59, 2490 (1997).

[41] Z.-C. Yan and G. W. F. Drake, Phys. Rev. A 52, 3711 (1995).

[42] K. T. Chung, Phys. Rev. A 44, 5421 (1991); 45, 7766 (1992). K. T. Chung and X.-W. Zhu, Phys. Scr. 48, 292 (1993).

[43] J. Pipin and D. M. Bishop, Phys. Rev. A 45, 2736 (1992).

[44] M. Tong, P. Jonsson, and C. F. Fischer, Phys. Scr. 48, 446 (1993).

[45] G. Pestka and W. Woznicki, Chem. Phys. Lett. 255, 281 (1996).

[46] I. Sakho. Chinese journal of physics vol. 48, no. 5 (2010). 